\title{
APORTACIONES AL COMPORTAMIENTO RESISTENTE DE ESTRUCTURAS DE HORMIGÓN ARMADO AFECTADAS POR LA CORROSIÓN DE SUS ARMADURAS
}

\section{(CONTRIBUTIONS TO THE ASSESSMENT OF LOAD-BEARING CAPACITY OF REINFORCED CONCRETE STRUCTURES WITH CORRODED REBARS)}

\author{
María Dolores García Alonso*, Dr. Arquitecto \\ Universidad Politécnica de Madrid \\ Escuela Técnica Superior de Arquitectura
}

ESPAÑA

\section{RESUMEN}

Lna de las principales causas que origina el deterioro prematuro de las estructuras de hormigón armado es la corrosión de las armaduras.

La presente Tesis aborda la incidencia de la corrosión en dos aspectos básicos del comportamiento estructural: las propiedades mecánicas del acero y la adherencia acero/ hormigón. Para ello, se desarrolla un programa experimental y un modelo teórico teniendo en cuenta los principales parámetros que intervienen en los temas estudiados.
Directores de Tesis: Jesús Rodríguez Santiago, Dr. Ingeniero de Caminos José Pedro Gutiérrez Jiménez, Dr. Ingeniero de Caminos

Fecha de recepción: 21-V-96

\section{SUMMARY}

Reinforcement corrosion is one of the main causes of the early deterioration of reinforced concrete structures.

This Doctoral Thesis deals with the consequences of corrosion on two basic aspects of the performance of structures: the mechanical properties of steel and steel-toconcrete bond. For this matter, an experimental program and a theorical model are developed taking into account the main parameters which influence the studied themes.

\section{Introducción}

En la actualidad, la corrosión de armaduras es uno de los mecanismos de deterioro que con más frecuencia se detecta en las estructuras de hormigón armado en servicio.

Cuando el agente agresivo, desencadenante de la corrosión, entra en contacto con la armadura, provocando la despasivación de la misma, se inicia un

* Tesis leida el 14 de noviembre de 1995 en la E.T.S. de Arquitectura de Madrid. Obtuvo la calificación de Apto Cum Laude por unamimidad. proceso de deterioro progresivo que afecta a las características de los materiales constitutivos del hormigón armado (acero y hormigón), así como a los mecanismos de interacción entre ambos (adherencia). En consecuencia, el comportamiento estructural del elemento dañado se verá afectado.

Por tanto, para desarrollar métodos de evaluación y recálculo de aplicación a estructuras afectadas por la corrosión de armaduras es necesario, y previo, analizar y cuantificar los daños causados sobre los materiales, acero y hormigón, y sobre el fenómeno que posibilita la acción compuesta entre ellos, la adherencia. 


\section{Objetivo del trabajo}

El objetivo de la Tesis ha sido evaluar los efectos de la corrosión en dos aspectos básicos del comportamiento resistente de estructuras de hormigón afectadas por este problema patológico. Estos aspectos son:

a) las propiedades mecánicas del acero

b) la adherencia acero/hormigón.

Para abordar el primer aspecto se planteó la realización de un programa experimental consistente en la ejecución de ensayos de tracción estándar con barras previamente corroídas mediante un proceso acelerado. Analizando la variación de los principales parámetros que informan sobre el comportamiento tensodeformacional del acero, en función del nivel de corrosión, sería posible cuantificar el deterioro de determinadas propiedades del material.

En cuanto al estudio de la influencia de la corrosión sobre la adherencia, el análisis se realizó para niveles de deterioro incipientes, es decir, desde que se inicia la corrosión hasta que el daño se manifiesta con fisuras de $0,3 / 0,4 \mathrm{~mm}$. Para ello se han seguido dos procesos diferentes:

* En primer lugar, mediante un modelo teórico, se aproxima la tensión de adherencia residual antes de que aparezcan las fisuras por corrosión.

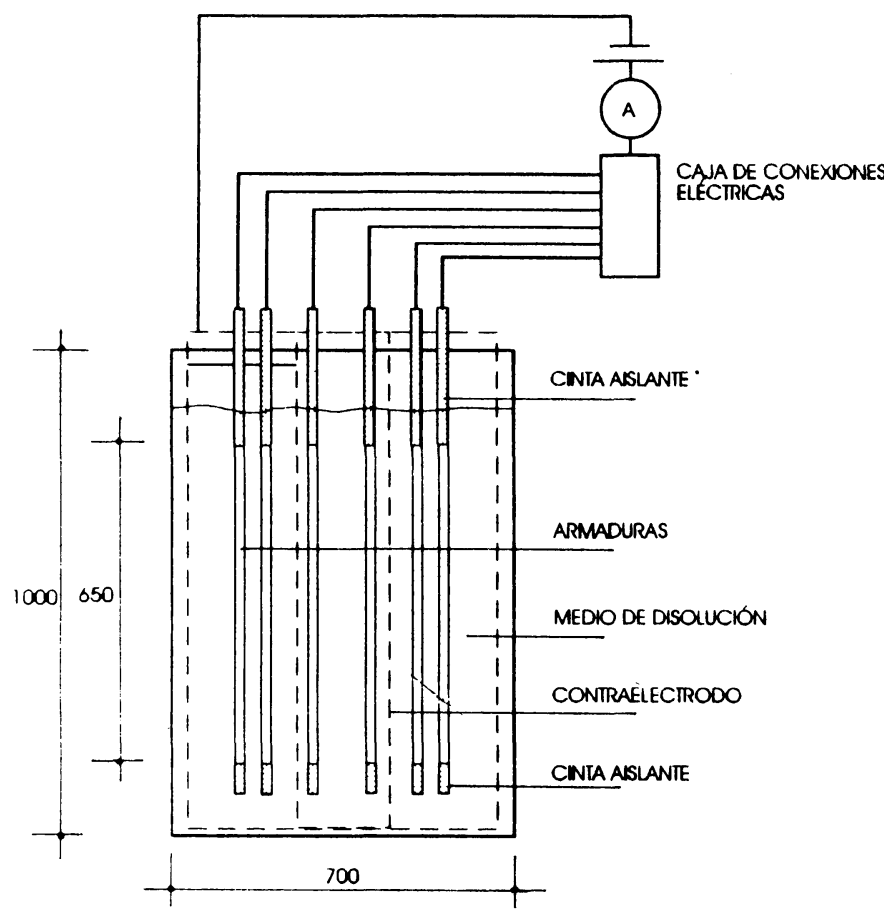

a) SECCIÓN
* En segundo lugar, se realiza una investigación experimental para estudiar la capacidad de adherencia una vez que la corrosión ha provocado fisuras longitudinales en el recubrimiento.

\section{Influencia de la corrosión en las propiedades mecánicas del acero}

Se ensayaron a tracción un total de 54 barras de acero AEH-500 S, de diámetros $12 \mathrm{~mm}$ y $20 \mathrm{~mm}$, y $1 \mathrm{~m}$ de longitud, de las cuales 48 se sometieron a un proceso de corrosión acelerada. El ataque de las muestras se realizó en dos medios de disolución (agua potable y agua con $3 \%$ en peso de $\mathrm{NaCl}$ ) para simular las causas más comunes que desencadenan la corrosión de armaduras embebidas en hormigón: la carbonatación y la presencia de cloruros.

Para corroer las barras se utilizó un método galvanostático, aplicando a las muestras una corriente eléctrica del orden de $1 \mathrm{~mA} / \mathrm{cm}^{2}$, una vez que estaban sumergidas en el electrólito de ensayo (figura 1). Previamente se realizaron una serie de pruebas, a pequeña escala, que permitieron eliminar ciertas reservas para extrapolar los resultados de los ensayos acelerados a los procesos de corrosión espontáneos.

Una vez que se terminó la corrosión acelerada de las muestras se obtuvieron las pérdidas gravimétricas generadas en el proceso. A partir de las mismas se

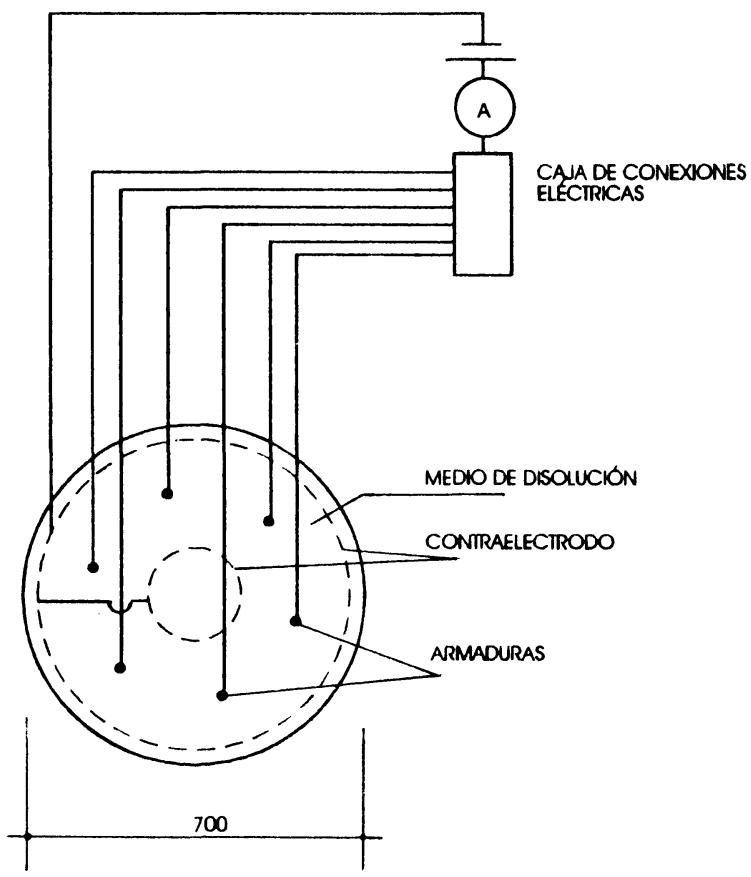

b) PLANTA

Fig. l.- Esquema del dispositivo utilizado para provocar la corrosión acelerada de las armaduras. 
calcularon los valores de reducción de sección y profundidad de ataque que se tomaron como referencia para analizar los resultados.

Los ensayos de tracción permitieron evaluar, en función del nivel de corrosión alcanzado, la variación de los principales parámetros que informan de la resistencia y ductilidad del acero: límite elástico, resistencia a tracción, deformación tras la rotura y deformación bajo carga máxima.

Los resultados mostraron que la influencia de la corrosión en los parámetros tensionales, límite elástico $f_{s}$ y resistencia a tracción $f_{s u}$, es muy poco significativa para niveles de daño inferiores al $20 \%$ de pérdida de sección, que cubren ampliamente muchos de los casos reales de corrosión generalizada. Niveles de deterioro mayores (del orden de $30 \%-40 \%$ de pérdida de sección) sí conllevan una reducción de $f_{s y}$ y $f_{s i}$.

En cuanto a la ductilidad, se obtuvo una disminución significativa de la deformación bajo carga máxima, que se puede cuantificar en torno al $20 \%$ para niveles de corrosión del $10 \%$ aproximadamente, y puede alcanzar un $40 \%$ para pérdidas de material iguales o superiores al $25 \%-30 \%$.

\section{Influencia de la corrosión en la adherencia acero/hormigón}

Como se ha comentado anteriormente, la investigación se aborda desde una doble vertiente:

- Por un lado, se hace una aproximación analítica para estimar la evolución de la adherencia antes de que el nivel de corrosión sea tal que provoque la aparición de fisuras en el exterior del recubrimiento.

- Por otro lado, se realiza una investigación experimental para estimar la capacidad de adherencia una vez provocadas las fisuras por corrosión.

\section{Modelo teórico}

El modelo teórico desarrollado en la Tesis permite estimar la capacidad de adherencia residual correspondiente a la fisuración, en función del nivel de corrosión. Para ello, se parte del hecho de que tanto las fuerzas de adherencia como la corrosión (los óxidos son más voluminosos que el acero del que proceden) ejercen una presión radial sobre el hormigón que rodea la armadura. En consecuencia, ambos fenómenos inducen la formación de anillos de tracción en el hormigón que equilibran dicha presión radial.

Por tanto, las componentes radiales de las fuerzas de adherencia y la presión ejercida por los productos de corrosión se pueden asimilar a una presión hidráulica actuando contra un anillo de pared gruesa, cuyo espesor será igual a la dimensión del menor recubrimiento.

En el análisis se establece una relación entre la presión y el desplazamiento radial en el interior del anillo, asimilable a la expansión impuesta por los productos de corrosión, hasta que se agota la capacidad portante del mismo. Se plantea una posible evolución del estado tensional y deformacional de la pared del anillo que sienta las bases del modelo propuesto. En el mismo se contemplan tres etapas: elástica, elastoplástica y elastoplástica con fisuración (figura 2).
1

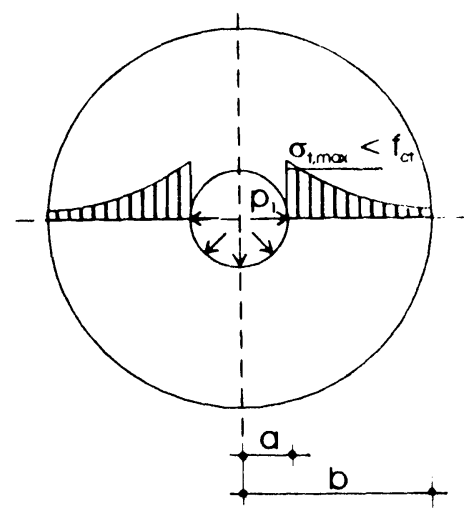

2

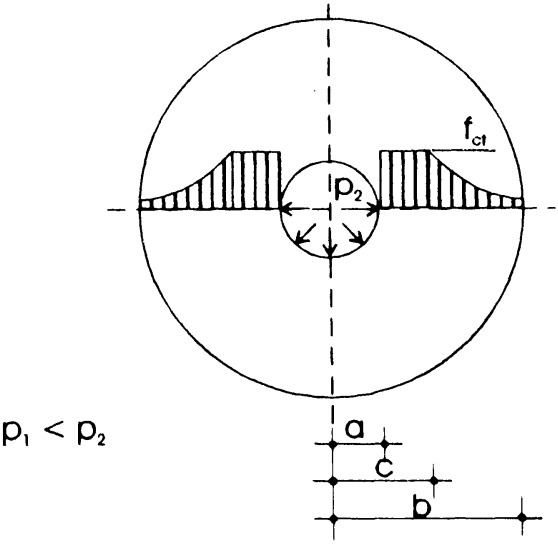

3

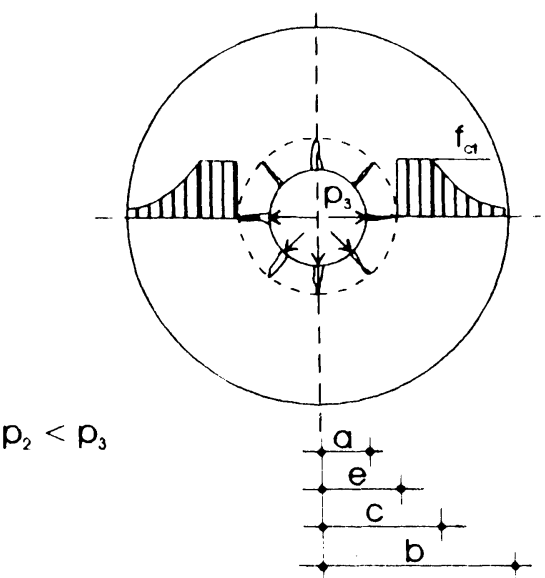

Fig. 2.- Distribución de tensiones de tracción en un anillo de pared gruesa sometido a una presión interna creciente. I) Etapa elástica; 2) Etapa elastoplástica; 3) Etapa elastoplástica con fisuración. 
Las hipótesis en las que se basa el modelo son:

- El hormigón es un material capaz de redistribuir tensiones de tracción desde las zonas más solicitadas a las menos solicitadas, de modo que se puede suponer una "plastificación completa" en longitudes cortas.

- La presión necesaria para fisurar el recubrimiento se fija en base a resultados experimentales, en ensayos de adherencia "Pull-Out", y en ensayos para caracterizar la relación corrosión/fisuración, realizados por otros autores.

- Una vez superada la presión máxima en el estado elástico, la plastificación avanzará desde el interior del anillo hacia el exterior a medida que aumenta la presión.

- Cuando el radio exterior del anillo plastificado sea tal que las deformaciones circunferenciales igualen la deformación máxima a tracción del hormigón $\left(\varepsilon_{t, \max }=\varepsilon_{r o t}\right)$, se iniciará una fisura interna. Sin embargo, esta fisura no atravesará la pared del anillo hasta que se agote la capacidad de carga del mismo.

- A partir de ese momento la plastificación y la fisuración avanzarán de modo que se cumpla $\varepsilon_{t, \max }$ $\varepsilon_{r o t}$ cte, y la rotura se producirá cuando se alcance la presión máxima para esa condición.

El modelo es válido para relaciones recubrimiento/ diámetro comprendidos entre 1 y 2,5 , que cubren ampliamente las relaciones usuales que se dan en estructuras de edificación.

También se ha considerado la posibilidad de disponer armadura transversal confinando el anillo de hormigón. En este caso, suponiendo compatibilidad de deformaciones entre el acero y el hormigón, la aportación de la armadura transversal se puede aproximar con bastante rigor, a partir de la expresión proporcionada por el análisis elástico.

\section{Investigación experimental}

El estudio del deterioro de la adherencia debido a la corrosión cuando ésta ha provocado la formación de fisuras longitudinales en el recubrimiento, se acometió mediante un programa de ensayos en los que la geometría de la probeta y la situación de las barras en ella reproducen situaciones que pueden darse en la práctica. En concreto, el tipo de ensayo de adherencia realizado en esta investigación simula las condiciones de un anclaje situado en la zona de una viga sometida a una solicitación de cortante constante (figura 3 ).

Se ensayaron un total de 27 probetas de $300 \mathrm{~mm}$ de ancho, $350 \mathrm{~mm}$ de alto y $350 \mathrm{~mm}$ de longitud. El recubrimiento, tanto horizontal como vertical, fue de $30 \mathrm{~mm}$ y el diámetro de las armaduras $20 \mathrm{~mm}$, dando en todas las muestras una relación recubrimiento/diámetro $=1,5$.

Las armaduras longitudinales de 18 probetas se sometieron a un proceso de corrosión acelerada. Para ello, al fabricar el hormigón se añadió en el agua de amasado un $3 \%$ de $\mathrm{CaCl}$ en relación al peso de cemento $\mathrm{y}$, posteriormente, se aplicó una corriente eléctrica a cada una de las armaduras del orden de $100 \mu \mathrm{A} / \mathrm{cm}^{2}$. Este valor de densidad de corriente es 10 veces mayor que las máximas velocidades de corrosión medidas en estructuras deterioradas en servicio, si bien en ensayos de laboratorio con hormigones muy contaminados de cloruros se han llegado a registrar valores de velocidad de corrosión similares al empleado en esta investigación.

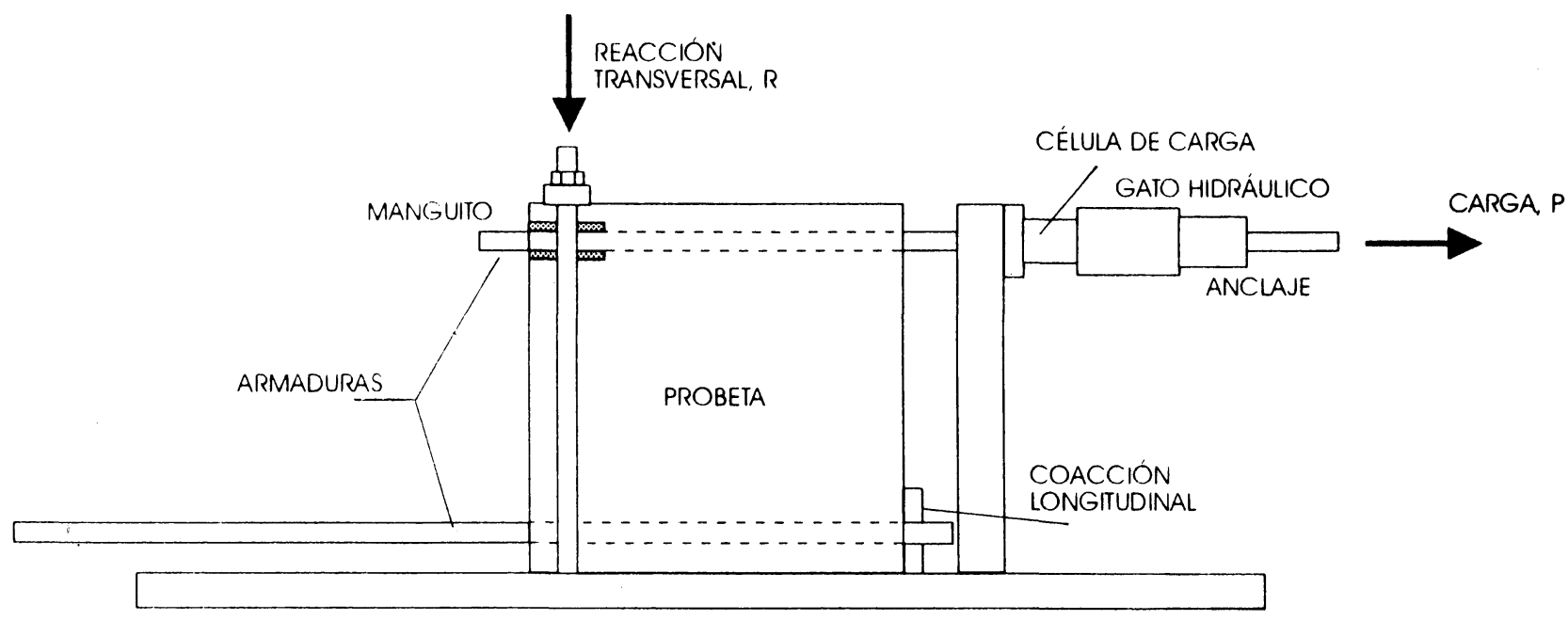

Fig. 3. - Esquema del tipo de ensayo de adherencia utilizado en la Tesis. 
Se realizaron ensayos con distintos niveles de corrosión, calculados a partir de la pérdidas gravimétricas generadas en el proceso, dentro de un rango de daño aparentemente poco importante (fisuras longitudinales de ancho medio entre $0.1 \mathrm{~mm}$ y $0.4 \mathrm{~mm}$, pérdidas de sección entre $1,5 \%$ y $5 \%$ y se contemplaron distintas cuantias de armadura transversal para verificar el efecto beneficioso que tiene sobre la adherencia (sin armadura transversal, $\approx c \phi 6 / 20 \mathrm{~cm}$ y $\approx c \phi 8 / 10 \mathrm{~cm}$ ).

Estos ensayos permitieron estudiar, en función del nivel de corrosión, la influencia de la posición de la barra durante el hormigonado (superior o inferior) y la influencia de la armadura transversal, para las cuantías empleadas

\section{Propuesta global}

A partir del modelo teórico y de la investigación experimental se hace una propuesta semicuantitativa para estimar la relación corrosión/resistencia de adherencia. Asi, la tensión última de adherencia asociada a un determinado nivel de corrosión se puede aproximar mediante la suma de la tensión remanente correspondiente al "splitting", proporcionada por el modelo teórico, y la tensión residual que aporta la armadura transversal, obtenida en la experimentación. Éste último valor es del orden de $2 \mathrm{MPa}$, no dependiendo de la calidad del hormigón ni de la posición de la barra durante el hormigonado.

En la figura 4 se ha representado la propuesta, considerando una relación recubrimiento/diámetro igual a 1.5 y una resistencia a tracción del hormigón $f_{c t}-2.4$ $M P a$.

\section{Agradecimientos}

La autora desea expresar su gratitud al Instituto de Ciencias de la Construcción Eduardo Torroja, donde ha sido desarrollada la Tesis, por facilitarle los medios materiales y humanos que han hecho posible el desarrollo de la investigación, y a todas las personas que, de una u otra forma, han contribuido a que este trabajo llegara a su fin.

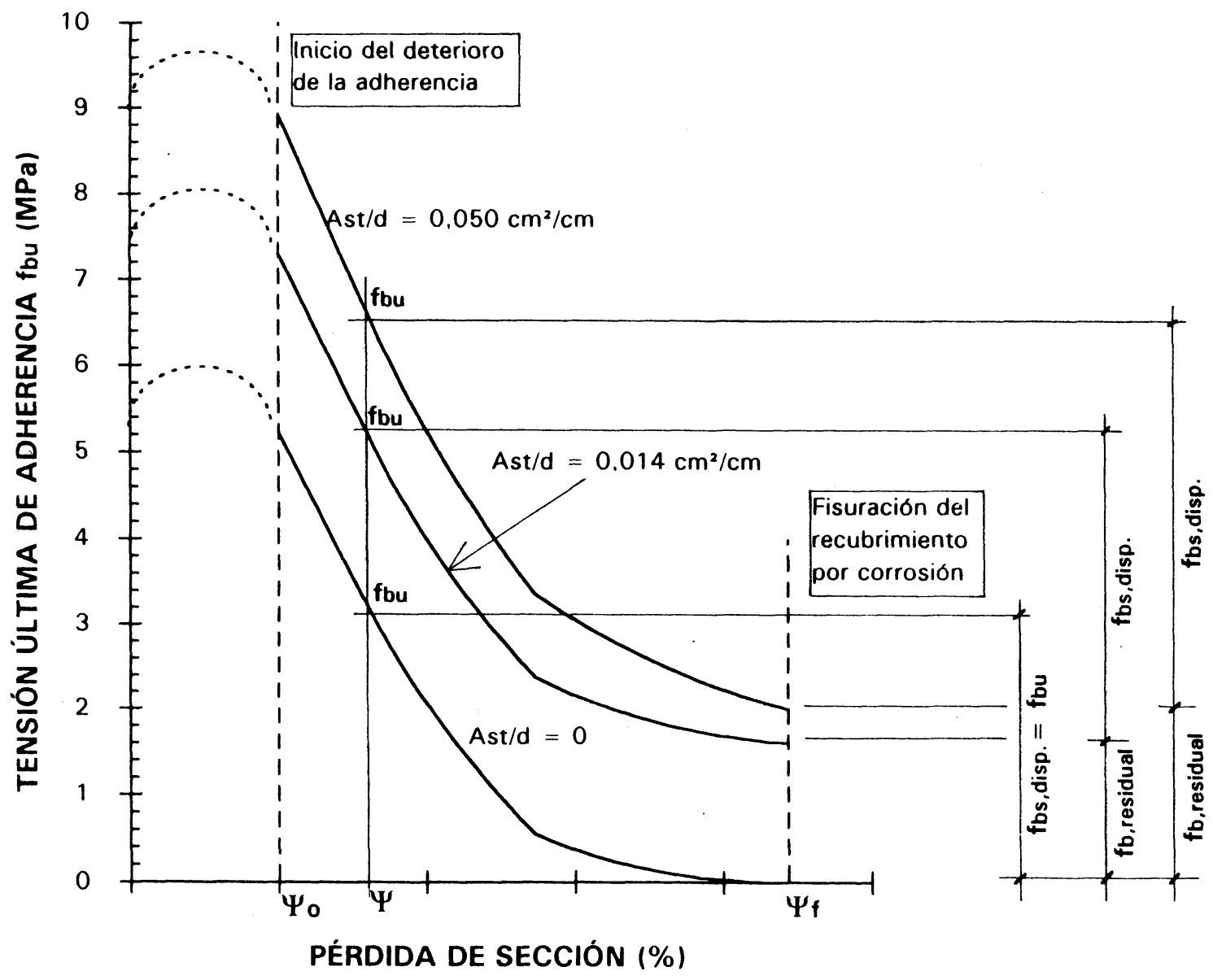

Fig. 4.- Representación del deterioro de la adherencia con la corrosión. 